\title{
Local Fiber Orientation from X-ray Region-of-Interest Computed Tomography of large Fiber Reinforced Composite Components
}

\author{
Thomas Baranowski ${ }^{\mathrm{a}}$, Dascha Dobrovolskij ${ }^{\mathrm{b}, \mathrm{c}}$, Kilian Dremel ${ }^{\mathrm{d}}$, Astrid \\ Hölzing ${ }^{\mathrm{d}}$, Günter Lohfink ${ }^{\mathrm{e}}$, Katja Schladitz ${ }^{* \mathrm{c}}$, and Simon Zabler ${ }^{\mathrm{d}}$

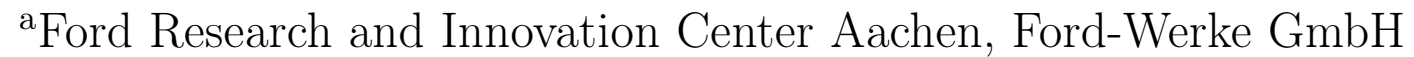 \\ ${ }^{b}$ Hochschule Darmstadt, Schöfferstraße 3, 64295 Darmstadt, Germany \\ ${ }^{\mathrm{c}}$ Fraunhofer-Institut für Techno-und Wirtschaftsmathematik, \\ Fraunhofer-Platz 1, 67663 Kaiserslautern, Germany \\ ${ }^{\mathrm{d}}$ Fraunhofer-Entwicklungszentrum Röntgentechnik, Josef-Martin-Weg \\ 63, 97074 Würzburg, Germany \\ eMontaplast GmbH, Morsbach, Germany
}

May 14, 2020

Keywords: non-destructive testing, glass fiber reinforced polymers, fiber orientation distribution, second order orientation tensor, injection molding

The local fiber orientation is a microstructural feature crucial for the mechanical properties of parts made from fiber reinforced polymers. It can be determined from micro-computed tomography data and subsequent quantitative analysis of the resulting 3D images. However, although being by nature non-destructive, this method so far

${ }^{*}$ Corresponding author:

katja.schladitz@itwm.fraunhofer.de has required to cut samples of a few millimeter edge length in order to achieve the high lateral resolution needed for the analysis.

Here, we report on the successful combination of region-of-interest scanning with structure texture orientation analysis rendering the above described approach truly non-destructive. Several regions of interest in a large bearing part from the automotive industry made of fiber reinforced polymer are scanned and analyzed. Differences of these regions with respect to local fiber orientation are quantified. Moreover, consistency of the analysis based on scans at 
varying lateral resolutions is proved. Finally, measured and numerically simulated orientation tensors are compared for one of the regions.

\section{Introduction}

Many structural components in the automotive and aircraft industries are made from fiber reinforced plastic (FRP) composite material. The fibers can be made of glass, carbon, or other materials, they can be short cut, long or even continuous, of various thicknesses and volume concentrations. Typically, the fiber component comprises 10 to $35 \%$ of the volume for injection molded materials (e. g. in [5]) and up to 50$60 \%$ for laminar FRP (e. g. [36, 43, 21, 17]) and consists of 6 to $15 \mu \mathrm{m}$ thick carbon fiber bundles or 10 to $18 \mu \mathrm{m}$ thick glass fibers

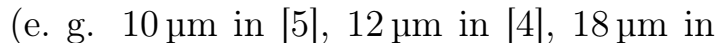
[20]).

When the components are molded, usually thermoplastics like polypropylene (e. g. [50]), polybutylene terephthalate (e. g. [4]), polyamide 66 (e. g. [6]), polyamide 6 , acrylonitrile butadiene styrene are used as host material to which the fibers are added. Since FRP components generally undergo mechanical and/or thermal stresses during their service life, their load bearing capacity/strength is of critical importance for the components' design.

The structural properties of injection molded FRP materials are locally anisotropic due to the fiber component being anisotropically oriented [1, 26, 6] (for short fibers), [18 (for long fibers). This anisotropy in turn is caused by the fibers moving with the liquid flow in the mold [8]. For complex shaped parts, the resulting fiber orientations are difficult to predict and control. As a consequence, structurally weak spots or areas can appear and may lead to early failure of the component. In order to avoid this, components are often designed too thick. This in turn thwarts the weight saving intention in using FRP.

Numerical simulations can predict the liquid flow [41] and thus indicate critical areas where material weaknesses might occur. Yet, these simulations are not perfect and need validation. Moreover, prediction of the local materials properties relies on orientation information as input, typically in the form of the 2nd order orientation tensor [1].

Fiber orientations can be analyzed essentially by four types of methods. Historically, before X-ray micro computed tomography $(\mu \mathrm{CT})$ became widely available, fiber orientations were evaluated through image analysis of polished 2D sections. See 45, Section 11.6.4] for a summary of stereological methods based on counting intersections in slices at varying angles and [28, 14] for orientations deduced from the shape of the observed elliptical cross-sections. These methods are not only destructive but the latter suffers also from the need for rather high resolutions and ambiguities due to the fact that there are always two 3D orientations generating the same cross-sectional ellipse. Therefore, critical areas of damaged/failed thermoplastic parts (or field returns) are nowadays predominantly analyzed by $\mathrm{CT}$ techniques, nevertheless for detailed verification optical microscopy of polished micro-sections is still commonly used.

X-ray micro computed tomography $(\mu \mathrm{CT})$ can be employed to analyze nondestructively the fiber orientation in structural components. Based on the resulting three-dimensional images, fiber orientations can be analyzed for the whole field of view (FoV) by the mean intercept length (MIL, [8]) or by measuring the 
length of generalized projections and obtaining the orientation distribution via the inverse cosine transform [30, Section 5.4]. Both methods are designed to be applied to the whole FoV, they can however be localized by applying them to small subvolumes. Nevertheless, in order to get an orientation in each voxel, one would have to center the sub-volume in each voxel. While inverting the cosine transform faces numerical instabilities, the MIL method has been applied successfully, see [8] also for a comparison to analysis of $2 \mathrm{D}$ virtual slices from the CT data as well as of $2 \mathrm{D}$ images of polished surfaces.

Local fiber orientation can of course be determined via single fiber segmentation. These approaches are typically very demanding with respect to image quality (contrast as well as lateral resolution), fiber volume fraction and spatial arrangement of the fibers. On the other hand, if successful they yield additional valuable information, in particular the fiber length distribution [38, 46] or positions with respect to failure regions [24]. Single fiber segmentation usually relies on tracking fiber center lines or cross-sections from slice to slice [36, 17] or local approximation of fibers by line segments [15, 39], ellipsoids [3, or cylinders [44, 27, 32]. 3] reconnects fragments based on local orientation, while [44, 27] just use the fragment orientations. All these approaches have in common that they demand rather slow orientation changes within one fiber (see the detailed discussion in 24]) and the fiber diameter to be resolved by at least 8 voxels. Viguié [48] and Kronenberger 24] do not need the former but [48 thrives on high image quality as provided by tomography using synchrotron radiation and both rely on resolutions of 8 voxels per fiber diameter and more. Pinter [32] claims 5 voxels per diameter to be sufficient, whereas the efficiency of the circular voting filter drops significantly for the lower resolution of 3 voxels per fiber diameter. In [6], orientations of short fibers or clusters of them are used to derive the homogenized orthotropic behaviour for cuboidal sub-volumes for use in FEM simulations.

Here, we concentrate on estimation of the local fiber orientation in the sense of assigning an orientation vector to each voxel belonging to the fiber system without fiber separation. For this purpose, several methods based on local approximation of fibers by ellipsoids [37, 2, 52, and on local gray value derivatives of first 23 and second order [51] have been proposed. The first order gray value derivatives are subsumed into the so called structure tensor and are applied e. g. by [12 and in VG STUDIO MAX, see e. g. [7]. Note that the structure tensor is not the orientation tensor as described by [1] and given in Equation (1) below. The second order derivatives form the so called Hessian matrix and are applied e. g. in MAVI, see e. g. [50]. The rationale behind both methods is that locally the fiber orientation is the one in which gray values change or are curved the least. In [51, all four methods are compared comprehensively based on simulated single fibers with a diameter of 10 voxels. In applications, the derivative based methods usually use 2-4 voxels per diameter [20, 50, 51, 46] while [2] rather demands 10. An exception is [12] applying the structure tensor at fiber bundle instead of fiber level thus allowing for voxel sizes of $50 \mu \mathrm{m}$. In [34, the authors present a different technique, the dark field scanning, for derivation of fiber orientation results in FRP. This method allows for even coarser resolutions of $86 \mu \mathrm{m}$.

All local and single fiber based orienta- 


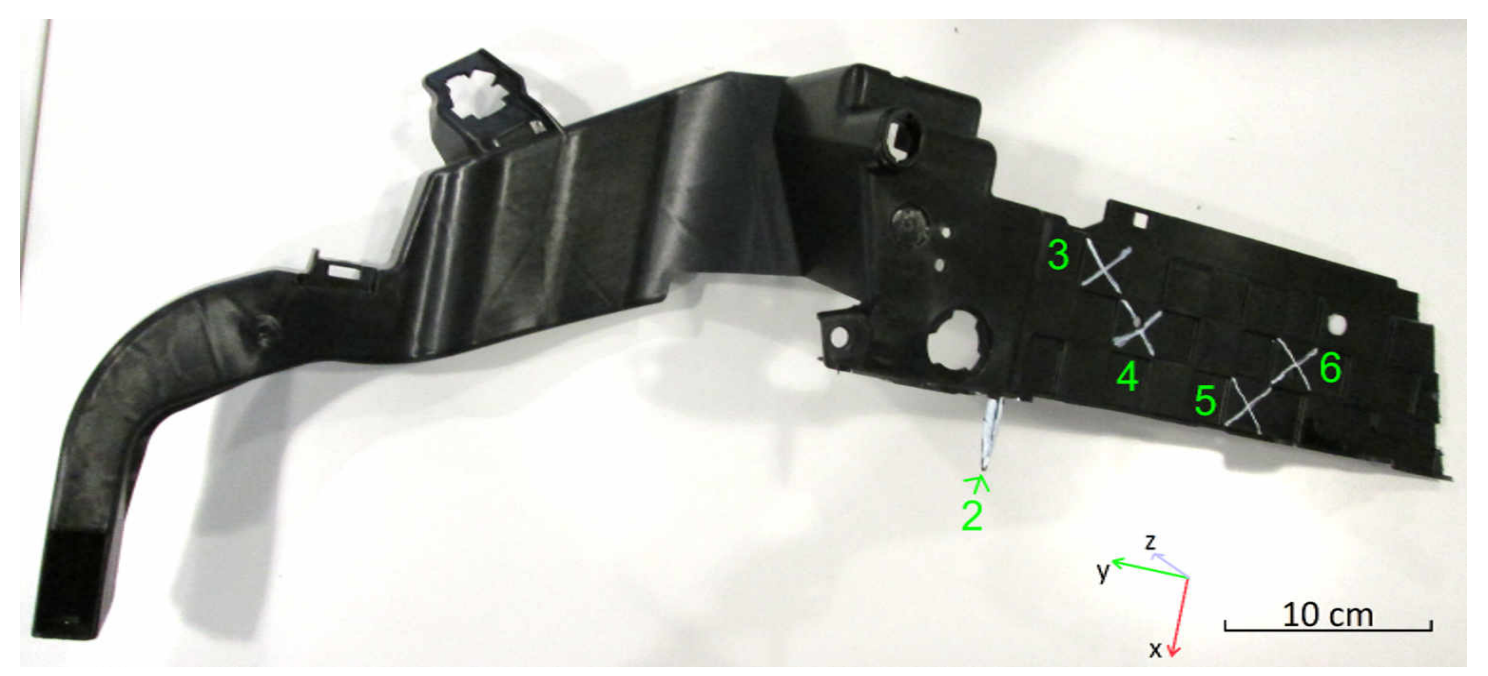

Figure 1: Bearing component made of polypropylene with $30 \mathrm{wt} \%$ reinforcing long glass fibers. Regions of interest (RoI) are marked white. As indicated, the analyzed component is mostly oriented in the $\mathrm{x}$-y-plane and positive $\mathrm{z}$ is the thickness direction. Throughout, this coordinate system is used.

tion analysis methods described above except [12, 34] require the fiber diameter to be sampled by at least 2-4 voxels [35, 50, 33. Sampling the fibers coarser than this causes them to crumble in the digital image. That is, a fiber sampled at less than 2 voxels for its diameter is endangered to form more than one connected component. Thus, similar to the microscopic imaging of planar sections $-\mu \mathrm{CT}$ is limited in its FoV to some $\mathrm{mm}^{3}$. E. g., if glass fibers of diameter $10 \mu \mathrm{m}$ are analyzed, orientation analysis limits the voxel size to at most $5 \mathrm{\mu m}$ and consequently the FoV to $10 \mu \mathrm{m} / 3 \times 2048 \approx 7 \mathrm{~mm}$ size. This FoV usually covers the component's thickness. However, the lateral dimensions of injection molded parts can be as large as some meters. So far, we used $\mathrm{CT}$ to analyze fiber orientations in small molded parts. These results have been used to verify simulation results of injection molding simulation software. Large molded parts could not be analyzed due to the limitations of $\mathrm{CT}$ devices used in the polymer industry. The part analyzed in this paper, shown in Figure 1, is about $1 \mathrm{~m}$ long.

As a consequence, very often the sample size is reduced to match the FoV determined by the needed resolution by cutting small pieces of a few millimeters edge length from the part thereby rendering $\mu \mathrm{CT}$ an utterly destructive inspection technique [53, 6].

With this work we demonstrate two strategies which aim at employing $\mu \mathrm{CT}$ for fully non-destructive analysis of local fiber orientation in injection molding FRP parts:

1. The imaging technique is applied as region of interest CT (RoI CT) [19]. That is, the components are not cut and the FoV can be significantly smaller than the components size, at least in one dimension.

2. Instead of the local fiber orientation distribution, a local "texture orientation" is derived. More precisely, the local orientation is determined in small 
cubic sub-volumes (boxes). Boxes not containing enough voxels belonging to the fiber component are not taken into account. Thus, the spatial sampling of the fiber diameter can be reduced to less than one voxel, allowing for an FoV 10 times larger than required for a local fiber orientation analysis.

By combining these two aspects into one novel method the analysis of local fiber orientation in meter-sized glass FRP components becomes readily feasible.

While in synchrotron $\mu \mathrm{CT}$ experiments RoI (axial or laminography) scans are performed routinely on carbon FRP material [9] this acquisition mode is still new in laboratory $\mu \mathrm{CT}$. One reason for this is the compactness of commercial $\mu \mathrm{CT}$ scanners which, given a high geometric magnification, leave little to no space between X-ray source window and sample. The present scanner uses a variable source-detector distance which allows for RoI CT in samples of $100 \mathrm{~mm}$ width while maintaining a high magnification and microscopic voxel sampling.

\section{Materials and methods}

The object used to demonstrate our technique is a long glass fiber composite carrier, see Figure 1. Built into the upper front end of the car, this part fulfills several functions: It carries the hood damper and yields mounting points for the lights as well as for the radiator package. The latter is provided with air by the carrier, too. Moreover, jointly with the crash absorber, the carrier contributes to fulfilling the legal regulations w.r.t. pedestrian protection.

In a polypropylene matrix, $30 \mathrm{wt} \%$ glass fibers of $10-20 \mu \mathrm{m}$ thickness and $10-15 \mathrm{~mm}$ length before processing are embedded.
The carrier has been scanned several times with different parameters. Thus, data has been acquired at several resolutions revealing different local features of the particular regions. The scanned regions were chosen to evaluate the effect of flow on fiber orientations along the part. Table 1 summarizes physical and digital sizes of the scans. The largest analyzed RoI scan A3.1 was scanned at the coarse resolution of $44 \mu \mathrm{m} /$ voxel edge by the Tomosynthesis scanner at a the Fraunhofer Institute in Fürth (EZRT), Germany. This scanner has a maximum source-detector distance of $2 \mathrm{~m}$, while its detector has $100 \mu \mathrm{m}$ pixel pitch. Thus the scanner does not allow for very fine voxel samplings $(<5 \mu \mathrm{m})$ while maintaining a large source-object distance (in the present case at least $100 \mathrm{~mm}$ ). We therefore used the metRIC scanner at EZRT Würzburg for the remaining scans. The metRIC allows for voxel samplings down to $2.33 \mu \mathrm{m}$ with $100 \mathrm{~mm}$ sourceobject distance thanks to its very large detector $\mathrm{X}$-axis (up to $3.3 \mathrm{~m}$ ) and a pixel pitch of $74.8 \mu \mathrm{m}$. Thus, metRIC allows for higher resolutions while coping with large size of the scanned component. So that, we scanned the RoI A2, A4 and A5 at higher resolutions of $10-20 \mu \mathrm{m} /$ voxel edge. In order to compare the local analysis at several resolutions, the region $\mathrm{A} 3.2$, has been imaged at coarse $(45 \mu \mathrm{m} /$ voxel, A3.2m), intermediate $(21 \mu \mathrm{m} /$ voxel, A3.2h), and high resolutions $(10 \mu \mathrm{m} /$ voxel, A3.2uh). The scans A3.2m, A3.2h and A3.2uh have been acquired by changing exclusively the source-object-distance/source-detectordistance leading to the corresponding dimensions of the scanned RoI. Finally, RoI A3.3 has been scanned at the highest resolution of $3 \mu \mathrm{m} /$ voxel edge. Next, we describe the CT-data acquisition set-ups. Afterwards, we present the orientation anal- 


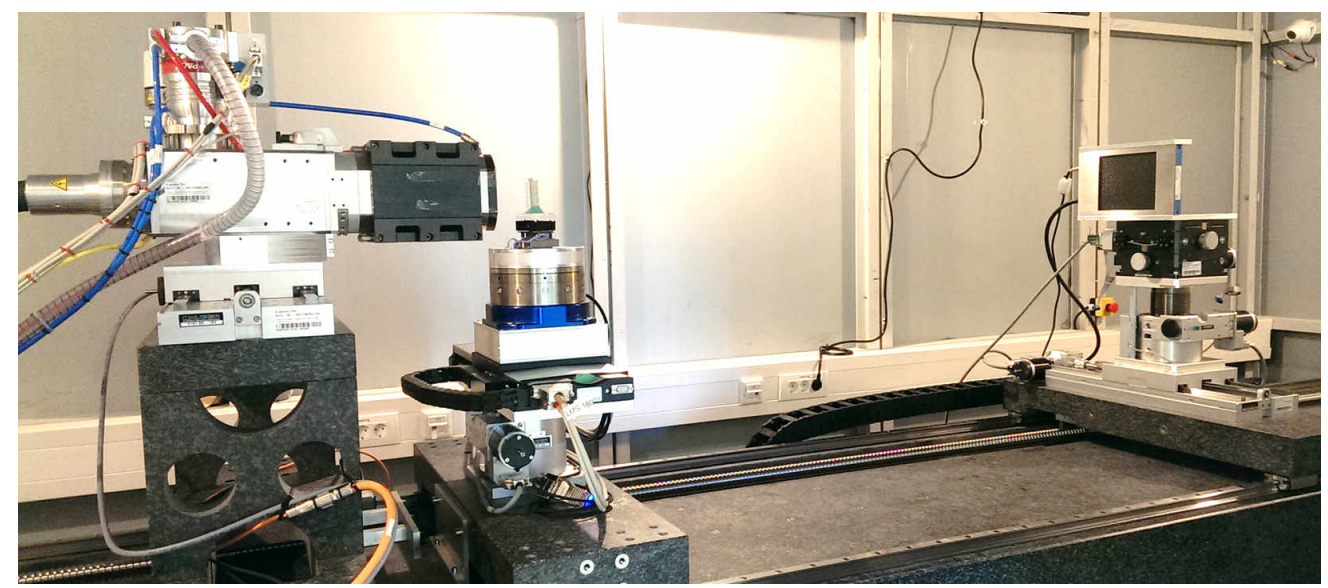

Figure 2: RoI CT scanner MetRIC at EZRT in Würzburg.

ysis results for the CT-data and compare them with Moldflow ${ }^{\circledR}$ simulations.

\subsection{X-ray approach for entire components (Rol CT)}

RoI CT of large glass FRP components requires certain degrees of freedom in the $\mathrm{CT}$ scanner as well as sufficient space for displacement of the sample. This is realized in the Tomosynthesis scanner. The scanner allows for precise $\mathrm{x}, \mathrm{y}$, and $\mathrm{z}$ movements over more than one meter range, thereby placing FoV at any position and of variable size and detail between X-ray source and detector. The X-ray source is an open microfocal transmission anode which provides $\mathrm{X}$-ray spot sizes down to $1 \mu \mathrm{m}$. The Xray projections of the sample are recorded on a digital detector array which covers an area of $40 \mathrm{~cm} \times 40 \mathrm{~cm}$ (Varian PaxScan). The sample is mounted vertically on the object table and the RoI is positioned on the marked positions covering $5 \mathrm{~cm}$ FoV which are sampled at $22.4 \mu \mathrm{m} /$ voxel (geometric magnification $4.45 \times$ ). Binning of two detector pixels results in the final effective voxel edge length of $45 \mu \mathrm{m}$. Reconstruc- tion of the volume images from 3000 projections is achieved through standard Feldkamp back-projection.

In addition to the low and medium resolution scans of RoI A2, A3.1, A3.2, A4 and A5, we applied high resolution local tomography to the same sample. The RoI A3.3 covers the region marked by the elongated red box in Figure 3. The RoI CT scanner MetRIC (see Figure 2) has been designed and constructed recently at the EZRT in Würzburg, Germany. The X-ray source is an X-RAY WorX microfocus transmission anode (XWT-190-THCE PLUS) which can be operated at up to $190 \mathrm{kV}$ acceleration voltage and provides a spot size down to below $1 \mu \mathrm{m}$ with the high-resolution target ( $1 \mu \mathrm{m} \mathrm{W}$ on $250 \mu \mathrm{m} \mathrm{Be})$. The setup comprising source, sample manipulator, and detector offers 10 degrees of freedom and a precisely encoded positioning of the sample and probed RoI. The flat-panel detector (Perkin Elmer Dexela 1512 NDT, 14bit CMOS, $\mathrm{Gd}_{2} \mathrm{O}_{2} \mathrm{~S}$ :Tb DRZS-scintillation screen) features a pixel size of $74.8 \mu \mathrm{m}$ on a sensitive area of $154.4 \mathrm{~mm} \times 114.9 \mathrm{~mm}$. Especially the extended movement of up to 


\begin{tabular}{|c|c|c|c|r|}
\hline RoI & CT device & $\begin{array}{c}\text { dimensions in } \\
\text { x-y-plane }\left[\mathrm{cm}^{2}\right]\end{array}$ & voxels & $\begin{array}{r}\text { voxel size } \\
{[\mathrm{m}]}\end{array}$ \\
\hline \hline A2 & MetRIC & $2.7 \times 0.9$ & $604 \times 200 \times 205$ & 45 \\
\hline A3.1 & Tomosyn & $6.2 \times 4.8$ & $1422 \times 1102 \times 450$ & 44 \\
A3.2m & MetRIC & $4.9 \times 2.5$ & $1084 \times 556 \times 172$ & 45 \\
A3.2h & MetRIC & $2.0 \times 1.4$ & $949 \times 686 \times 307$ & 21 \\
A3.2uh & MetRIC & $1.0 \times 0.7$ & $972 \times 726 \times 543$ & 10 \\
A3.3 & MetRIC & $0.6 \times 2.3$ & $1944 \times 8832 \times 1700$ & 3 \\
\hline A4 & MetRIC & $3.0 \times 1.9$ & $1860 \times 1150 \times 360$ & 17 \\
\hline A5 & MetRIC & $5.2 \times 5.6$ & $1210 \times 1300 \times 250$ & 44 \\
\hline
\end{tabular}

Table 1: RoI scanned at varying resolutions.

$2.5 \mathrm{~m}$ of the detector stack along the horizontally oriented X-ray direction as well as of the X-ray source (up to $1.3 \mathrm{~m}$ ) enhances an optimized arrangement of encoded source, sample, and detector positions for well-balanced magnification (source-objectdistance/source-detector-distance), resolution and scanning time of each sample and RoI. The encoding of all axes enables CT scans without manual movement of the sample and the RoI can be reproduced at any time. The encoded $x-y$-stage on top of the rotary table as well as the z-movement of the sample stack allow for selection of several RoI without repositioning the sample. Consequently, all data is generated automatically in the same coordinate system.

Our scanner allows for an extensive focus-detector distance (here $3.3 \mathrm{~m}$ ) which in turn enables voxel samplings as small as $3 \mu \mathrm{m} /$ voxel, even in parts which are several $\mathrm{cm}$ wide. Moreover, on top of the air bearing rotation, a piezo positioning system lets the user choose an arbitrary RoI with sub$\mu \mathrm{m}$ precision.

The minimum resolution of all CT scanners depends on the sample size. Tomosynthesis as well as MetRIC enable resolutions down to 2-3 $\mu \mathrm{m}$. Tomosynthesis offers addi-

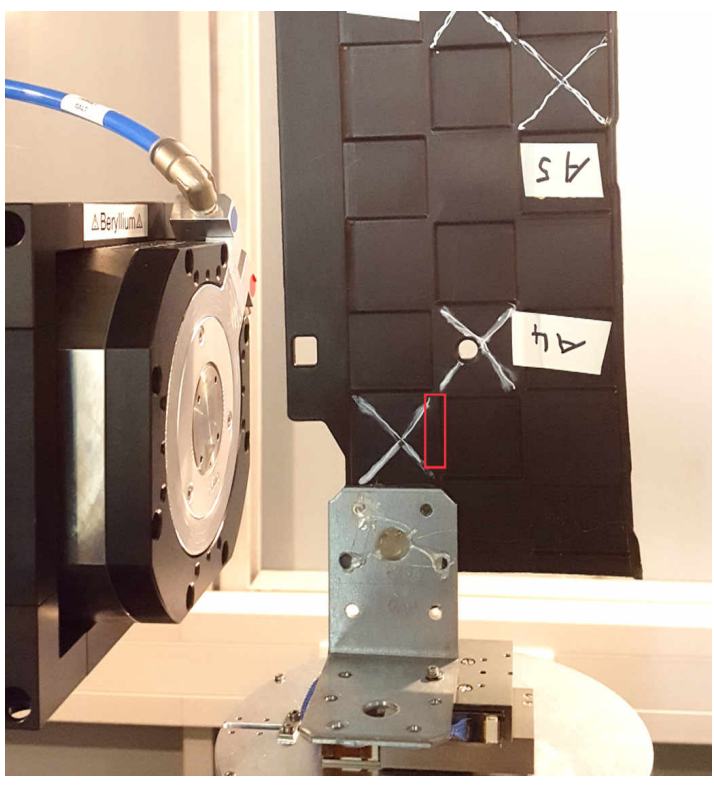

Figure 3: The red box marks the RoI which has been scanned at finest resolution, divided into six subvolumes. The stack of six scans covers approximately the entire red box. Figure 4(a) shows the volume rendering of the segmented fiber component of the whole RoI A3.3.

tionally the option to scan very large samples with high resolution. On the other 
hand, MetRIC has a smaller focal spot size reaching even higher resolutions for smaller samples. The degree of freedom for movements and total distances is higher and thus allows more variable sample geometries.

Although the 3D volume reconstruction of RoI CT data gives the chance to achieve high resolution images, it suffers from a few specific problems. If an area of the scanned object overlaps the reconstructable FoV in direction of the beam at a certain angle, this area will become part of the corresponding radiography. Therefore, in the image reconstruction process, this outer area will become part of the reconstructed volume. Moreover, problems occur when the projection of the scanned object is bigger than the horizontal detector size. Filtered back-projection of the radiographies involves high-pass filtering. As a consequence, the detector edge leads to reconstruction artifacts near the boundaries of the imaged volume.

Both problems are treated adequately at MetRIC. The scan is performed "on-thefly" using a non-stop rotational movement. That way, parts of the sample that are located outside the FoV will move faster during object rotation. Hence, the blurring of these areas is increased proportional to their distance to the center of rotation. In the resulting image slices, this leads to a constant gray value offset only. The horizontal overlap is handled by padding as the high-pass filter used for backprojection is less sensitive of the projected image. Thus, cupping artifacts in the outer areas of the reconstructed slices are avoided.

\subsection{Measuring local fiber orientation from 3D image data}

In this paragraph, we shortly summarize the method for local 3D fiber orientation analysis based on 2nd order gray value derivatives. That is, the method based on an eigenvalue analysis of the Hessian matrix in each voxel of a 3D image.

State of the art methods for analyzing the fiber orientation in $\mu \mathrm{CT}$ images of $\mathrm{FRP}$ parts determine the fiber orientation in each voxel without need to identify individual fibers [23, 33, 51, 13]. More precisely, in each voxel belonging to the fiber system, a local fiber orientation is derived. These voxel-wise measurements yield the volume weighted orientation distribution of the fiber system observed in the 3D image. As discussed in the Introduction, in general, segmenting individual fibers requires higher resolutions than the voxel-wise orientation analysis. The latter being reported to work at spatial sampling of the fiber diameter by 2-3 voxels ([35, 50, 33]), allows for an FoV considerably larger than required for single fiber analysis.

Here however, in the coarser scans, the mean fiber diameter of approximately $10 \mu \mathrm{m}$ is sampled by less than one voxel. Due to the local orientations not rapidly changing spatially, local orientation analysis is nevertheless possible, see [40] analyzing bundles of glass fibers in sheet molding compound samples at a nominal resolution of $17.3 \mu \mathrm{m}$ in virtual $2 \mathrm{D}$ slices using the method from [37] and [12] applying the structure tensor [23] to prepreg platelet compression molded samples for 3D orientation analysis at $50 \mu \mathrm{m}$ nominal resolution. The gray value of an image voxel represents in that case the averaged energy absorbed by several neighboring fibers.

\subsection{Local fiber orientation analysis}

The regions scanned are not simply cuboidal. Thus, first of all, masks for the RoI of the part are derived from the CT im- 
age data. To this end, solid matter is separated from the surrounding air by a manually chosen global gray value threshold. The resulting rough edges are smoothed by a morphological opening with a $3 \times 3 \times 3$ voxel cube.

The local orientations are measured in each voxel exploiting the second order partial derivatives of the local gray values. That is, the Hessian matrix is computed in each voxel. Subsequent analysis of the eigenvalues of the Hessian yields the local orientation of bright locally fiber like structures as the eigenvector corresponding to the smallest (in magnitude) eigenvalue. Following [51], we define a fiber like structure to be a subset of a dilated random fiber system which in turn is a collection of rectifiable curves. See [25, 29] for mathematical background. The idea behind the eigenvalue analysis is that standing on a (gray value) mountain ridge, the orientation of the ridge is the one in which the (gray value) relief is curved the least [16]. In [51], this method has been proven to be equivalent to the structure tensor based one of [23] and to outperform methods discretizing the orientation space, namely orientation derived from maximal response of anisotropic Gaussian filters [37] or from the moments of intertia [2].

Here, the Hessian matrix based method is slightly altered. In [51], the fiber diameter is assumed to be known. Calculation of the 2nd order partial gray value derivatives in each voxel is proceeded by smoothing with a Gaussian filter whose parameter is chosen to meet exactly the fiber radius. This choice is motivated by the interpretation of a bright glass fiber within a darker matrix forming a ridge in the gray value relief and the desire to observe the highest points of the ridge exactly at the center line of the fiber. Recent experiments [13] shed some doubt on this empirically deduced rule of thumb and this issue is currently being investigated. Clearly, choosing the width of the Gaussian as the fiber diameter is impossible if the diameter is resolved with less than 3 voxels. Thus, in these cases, a minimal smoothing filter with a $3 \times 3 \times 3$ voxel mask approximating a Gaussian is used.

The presented local orientation analysis is based on the mathematical concept of the typical point of a random closed set [30, 42, 45]. Very roughly speaking, one looks at the world from a point chosen "uniformly" within the random set. As long as the fibers are of equal thickness and do not intersect, the resulting distribution of the fiber orientation in this typical point is the same as if just the one-dimensional fiber cores are taken into account. Let $R$ be the distribution of the local fiber orientation in the typical point. That is, $R$ is a probability measure on the space of direction - the upper half-sphere $S_{+}^{2}$. The 2 nd order orientation tensor [1] can be interpreted as the 2nd moment of $R$. Let $u_{i}, \quad i \in\{x, y, z\}$ denote the component of some normalized direction vector $u$ in coordinate direction $i$. Then the second order orientation tensor is defined as $\left(a_{i j}\right)$ with

$$
a_{i j}=\int_{S_{+}^{2}} u_{i} u_{j} R(d u), \quad i, j \in\{x, y, z\}
$$

see [51.

Finally, the local orientation information is exploited for those voxels assigned to the fiber system by a global gray value threshold, only. This threshold is found by multiplying Otsu's threshold 31 by 1.25 , the rationale behind that being that the observed orientation distribution is not distorted if voxels at the fiber edges are systematically not taken into account.

An eigenvalue analysis for the 2nd order 
orientation tensor yields the preferred local direction as the eigenvector to the largest eigenvalue $\ell_{\max }$ as well as an index reflecting the strength of anisotropy, see [40]. More precisely, consider $\alpha=1-\ell_{\min } / \ell_{\max }$, where $\ell_{\min }$ is the smallest eigenvalue of the orientation tensor. This index assumes values in the range $[0,1]$ with 0 indicating perfect isotropy. A value of 1 is achieved in two cases - perfect unidirectional fibers or a transversally isotropic fiber system. Tensile tests for glass FRP reported in [40] suggest that samples with $\alpha<0.6$ behave as isotropic samples. Thus calculating a mean fiber direction is not sensible in this case.

\section{Results}

Here, we present first the results of the RoI CT scans by volume renderings and slice views in Section 3.1. The following Section 3.2 contains the 3D orientation analysis results, both locally as well as averaged for each region. Finally, for regions A3.2 and A3.3, local fiber orientations deduced from the image data are compared for several resolutions in Section 3.3 and for A3.3 to those obtained by numerical simulation of the injection molding process, see Section 3.4 .

All orientations are analyzed in $3 \mathrm{D}$, in each voxel, see [35]. There, the Hessian matrix based method is applied to data of FRP samples showing pixel-wise orientation results. In [49, the authors discussed the existing problems and challenges around the visualization of volumetric microstructures by means of FRP. Size and complexity of the imaged regions necessitate a reduction of orientation information. Here, we concentrate on the orientation tensor diagonal element $a_{y y}$ as the $y$-direction is the dominating one. Therefore, results are averaged in cubic sub-volumes and often 2D slices or projections are chosen for better illustration. Sub-volume sizes are nevertheless chosen with particular attention to formed layers, so called shell and core layers see [22, 10, 11], in the components' microstructure.

\subsection{Rol CT scans}

Altogether, four regions are scanned. Region A3 is scanned five times with A3.1, $\mathrm{A} 3.2 \mathrm{~m}, \mathrm{~A} 3.2 \mathrm{~h}, \mathrm{~A} 3.2 \mathrm{uh}$, and A3.3 being ordered w.r.t. ascending resolution. See Table 1 for dimensions and voxel sizes.

Figures 445 give a visual impression of a representative selection of the analyzed scans. Volume renderings and 2D slices of RoI A3.2uh, A4 and A3.1 reveal the differences in acquired data caused by varying resolutions and the micro-structure in the imaged RoI respectively. The data sets are cascaded in Figures 4 5 starting at the finest resolution and getting coarser towards the bottom. Simultaneously this cascade emphasizes the differences in the sample volume covered by the RoI, too. The 2D slices in Figures 445 are taken from the central layer of the plate. All scans are subsequently analyzed. However, local orientation analysis results are visualized only for the selected regions marked yellow in Figures 445 .

\subsection{Local fiber orientation tensors from 3D image analysis}

The computed orientation results are summarized in Table 2, We derive the fiber component as described in Section 2.2. The orientation tensors are computed based on a tiling by cubes of size $218 \mu \mathrm{m} \times 218 \mu \mathrm{m} \times 218 \mu \mathrm{m}$. Clearly the $\mathrm{y}$ direction is preferred (long axis of the car- 


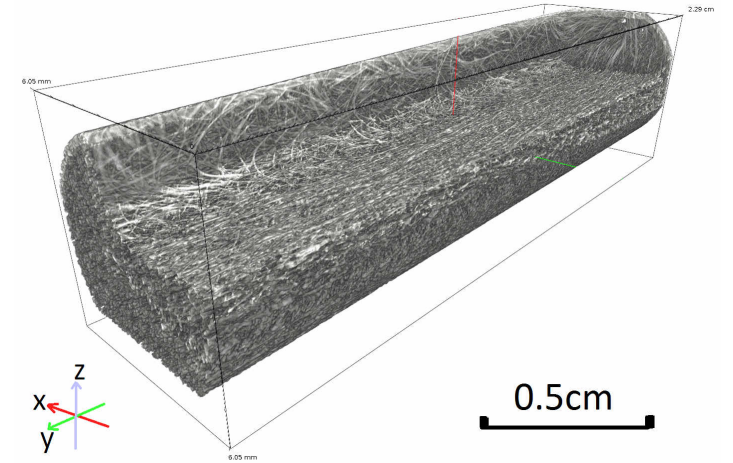

(a) A3.3, volume rendering

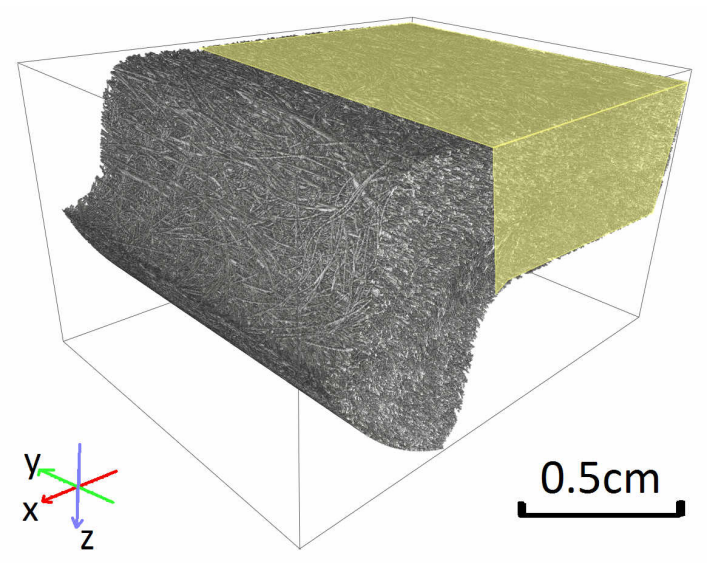

(c) A3.2uh, volume rendering

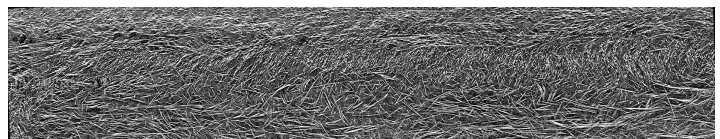

(b) A3.3, x-y-slice

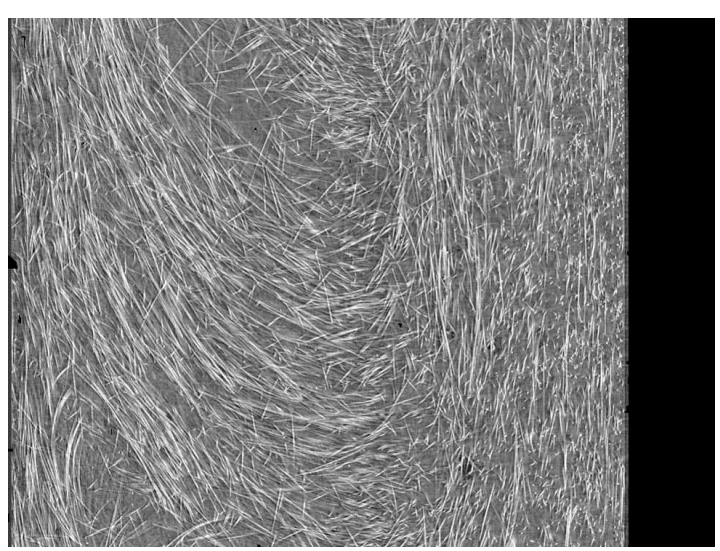

(d) A3.2uh, x-y-slice

Figure 4: Visualizations of the reconstructed CT images of RoI A3.3 and A3.2uh, with pixel sizes of 3 and $10 \mu \mathrm{m}$, respectively.

rier) for regions A3.1, A3.2, A3.3 and A5. In RoI A4, the y-direction is less dominant due to the reorientation around the hole in this region. RoI A2 is a special case as the component's shape differs significantly from the remaining plate-like shape in this spur region. A2 features $a_{x x}$ as the highest component. This finding is not surprising due to $\mathrm{x}$ being the longitudinal direction of the spur, see Figure 1 .

In the following Figure 6, we show the local orientation results for the regions marked yellow in Figures 445. The Figure shows slices from the upper (left) and central (right) layers of the plate like sub- regions. The color map visualizes the orientation tensor diagonal element $a_{y y}$ in flow direction y. For all resolutions, as expected, fiber orientations cluster around the flow direction in the upper layer and deviate stronger from this preferred direction in the central layer. Note that the orientation results in Table 2 are averaged over the entire regions. Thus, fibers being reoriented along the edges due to shaping are taken into account, too. This might decrease the anisotropy index as well as the dominating diagonal orientation tensor element. The yellow marked regions are tightly limited to the areas in plane and thus avoid the re- 


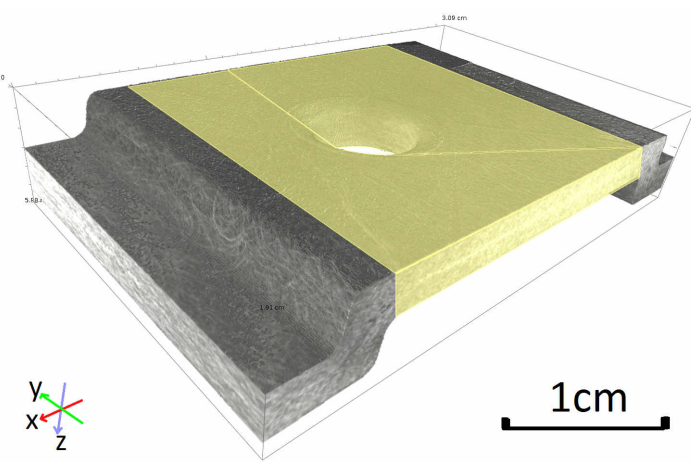

(a) A4, volume rendering

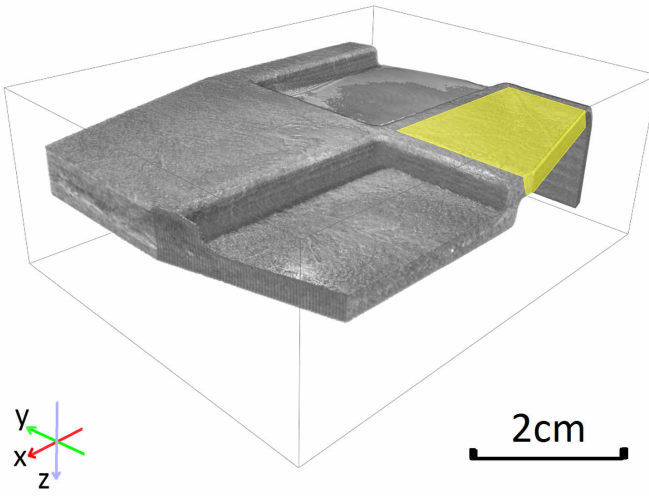

(c) A3.1, volume rendering

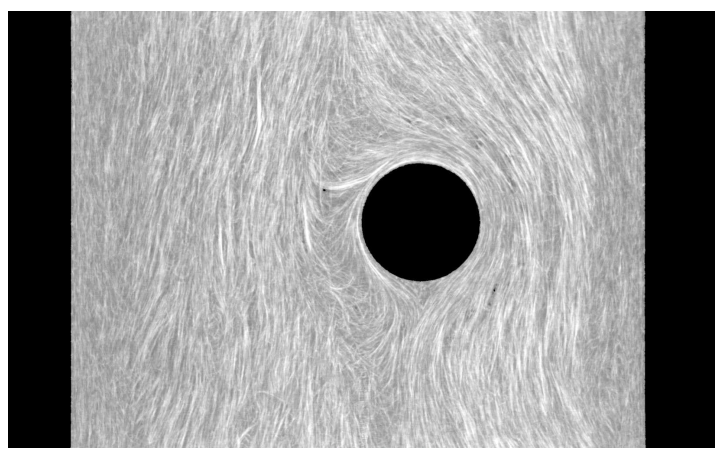

(b) A4, x-y-slice

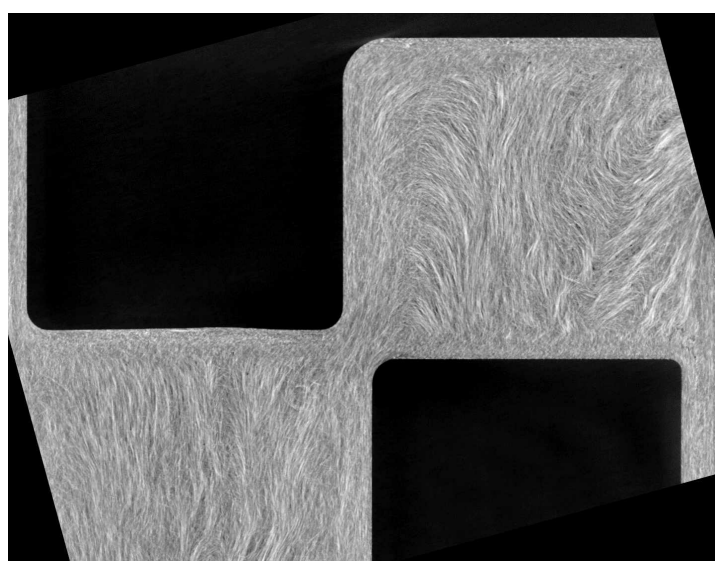

(d) A3.1, x-y-slice

Figure 5: Visualizations of the reconstructed CT images of RoI A4 and A3.1, with pixel sizes of 17 and $44 \mu \mathrm{m}$, respectively.

orientation of fibers due to shaping procedures. Orientation results for these regions are visualized in Figure 6 for the coarse resolution scans and the sequence of Figures 7 . 9 for the finest resolution scan.

Figures 7 and 8 show the 'edge' in region A3 which was scanned at the highest resolution $(3 \mu \mathrm{m} /$ voxel $)$. The orientation tensor is averaged in boxes of edge length $200 \mu \mathrm{m}$. The expected central layer deviating from the dominating y-orientation [4] is clearly visible in the rendering. Figure 9 shows the orientation tensor diagonal elements, averaged along the $\mathrm{x}$-axis of the shown volume. These graphs reveal that the central layer has a pronounced x-orientation $\left(a_{x x}\right.$ rising from 0.2 to approximately 0.5 along the entire strap) at the expense of y-alignment (dropping from 0.6 to 0.3 ) while the $a_{z z} \approx$ 0.2 tensor component is constant over the entire thickness (Figure 9(c)). Moreover, Figure 7 indicates a slight reorientation of fibers at one ending of the elongated volume. This visual impression is backed by Figure 9. Clearly, fibers are oriented mostly in plane in the first 120 sub-volumes along the y-axis in contrast to the last 15 subvolumes, where higher $a_{z z}$ values are observed. This is exactly where the fibers are reoriented due to shaping. 


\begin{tabular}{|c|c|c|c|c|}
\hline RoI & $\begin{array}{c}\text { mean fiber } \\
\text { direction }\end{array}$ & $\begin{array}{c}\text { anisotropy } \\
\text { index }\end{array}$ & $\begin{array}{c}\text { orientation tensor } \\
a_{x x}, a_{y y}, a_{z z}\end{array}$ & $\begin{array}{c}\text { voxel size } \\
{[\mu \mathrm{m}]}\end{array}$ \\
\hline \hline A2 & not applicable & 0.55 & $0.52,0.23,0.23$ & 45 \\
\hline A3.1 & not applicable & 0.55 & $0.25,0.51,0.23$ & 44 \\
A3.2m & $(-0.07, \quad 0.99,-0.00)^{T}$ & 0.61 & $0.23,0.54,0.21$ & 45 \\
A3.2h & $(-0.08, \quad 0.99,-0.02)^{T}$ & 0.60 & $0.22,0.54,0.22$ & 21 \\
A3.2uh & $(-0.06, \quad 0.99,-0.05)^{T}$ & 0.65 & $0.21,0.58,0.21$ & 10 \\
\hline A3.3 & $(-0.05,-0.99,-0.02)^{T}$ & 0.78 & $0.18,0.66,0.17$ & 3 \\
\hline A3.3.1 & $(-0.11,-0.99,0.03)^{T}$ & 0.75 & $0.18,0.63,0.18$ & 3 \\
A3.3.2 & $(-0.03,-0.99,-0.03)^{T}$ & 0.78 & $0.16,0.67,0.16$ & 3 \\
A3.3.3 & $(-0.08,-0.99,-0.04)^{T}$ & 0.76 & $0.18,0.64,0.16$ & 3 \\
A3.3.4 & $(-0.05,-0.99,-0.05)^{T}$ & 0.78 & $0.17,0.65,0.16$ & 3 \\
A3.3.5 & $(-0.04,-0.99,-0.04)^{T}$ & 0.77 & $0.18,0.63,0.17$ & 3 \\
A3.3.6 & $(0.01, \quad 0.99, \quad 0.04)^{T}$ & 0.77 & $0.19,0.63,0.17$ & 3 \\
\hline A4 & not applicable & 0.50 & $0.26,0.48,0.24$ & 17 \\
\hline A5 & not applicable & 0.57 & $0.22,0.51,0.25$ & 44 \\
\hline
\end{tabular}

Table 2: Orientation analysis results for the entire RoI, as specified in Table1. Note that the mean fiber direction is indicative only if the anisotropy index exceeds 0.6. The respective vectors are therefore not reported if the anisotropy is below this bound.

\subsection{Comparison of local fiber orientation tensors derived from scans at varying resolutions}

In order to compare the local orientations derived from scans at varying lateral resolutions quantitatively, we chose a sub-volume of A3 that is covered by several scans. More precisely, the chosen volume lies in the intersection of the RoIs A3.3, A3.2uh, A3.2h, and $\mathrm{A} 3.2 \mathrm{~m}$, scanned with voxel sizes 3$45 \mathrm{~mm}$. See Figure 10 for a volume rendering. We averaged the orientation results in sub-volumes of edge-length $200 \mu \mathrm{m}$ and subsequently along the $\mathrm{y}$-axis in order to preserve the characteristic differences between shell and core layers. Figure 11 shows the remarkable consistency of the orientation results even for the coarsest resolution at $45.3 \mu \mathrm{m}$. Nevertheless, the quality of ori- entation results drops for the coarser resolutions. This becomes obvious by the lower color contrasts between shell and core layers from left to right. Moreover, the component $a_{z z}$ orthogonal to the plate varies the most when resolved at $45.3 \mu \mathrm{m}$. These two observations clearly show a bias towards isotropy in the analysis results.

\subsection{Comparison to local fiber orientation tensors from injection molding simulation}

For three sub-regions of A3, we compare the 2nd order orientation tensors calculated in the previous section with the values obtained by Moldflow ${ }^{\circledR}$ simulations. The region is highlighted in yellow in Figure 12. The compared positions $\mathrm{p}_{1}$ to $\mathrm{p}_{3}$ are marked by triangles and rectangles in 


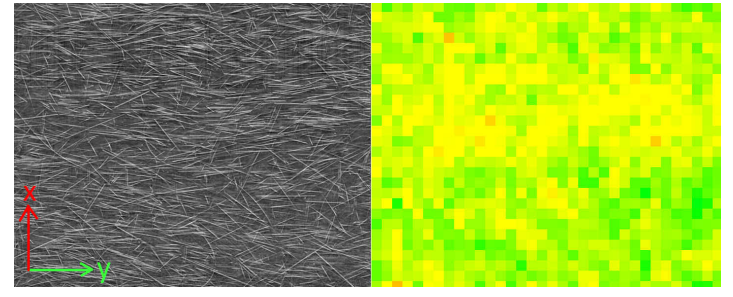

(a) A3.2uh, upper layer

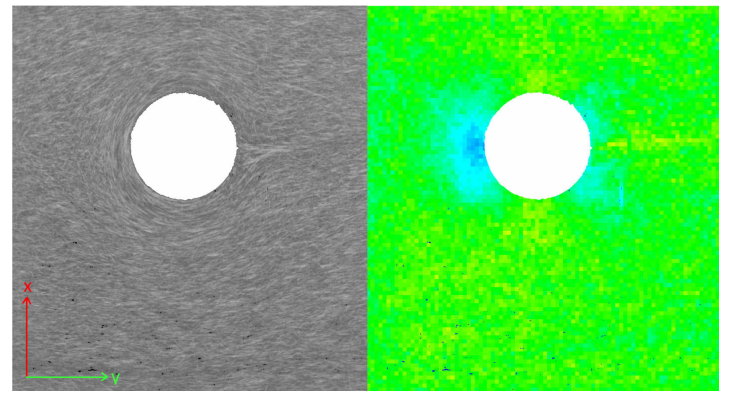

(c) A4, upper layer

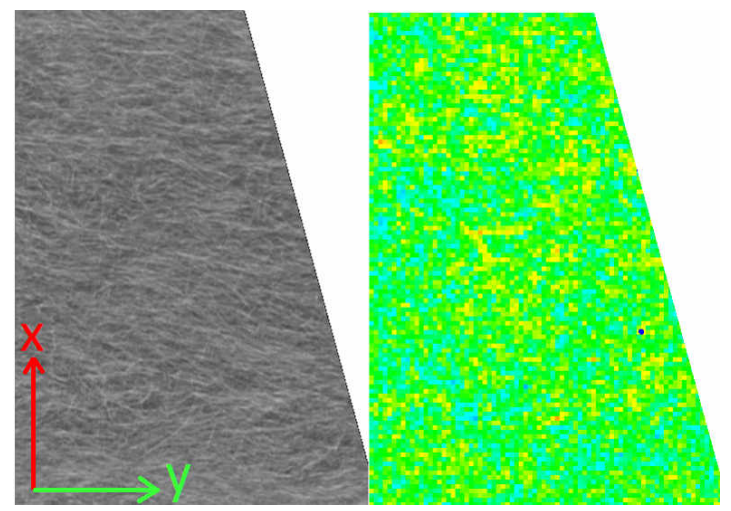

(e) sub-sample of A3.1, upper layer

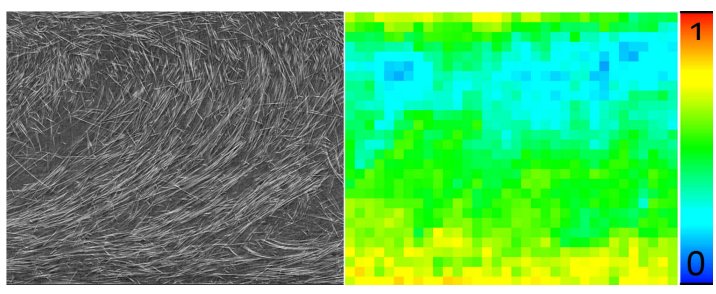

(b) A3.2uh, center layer

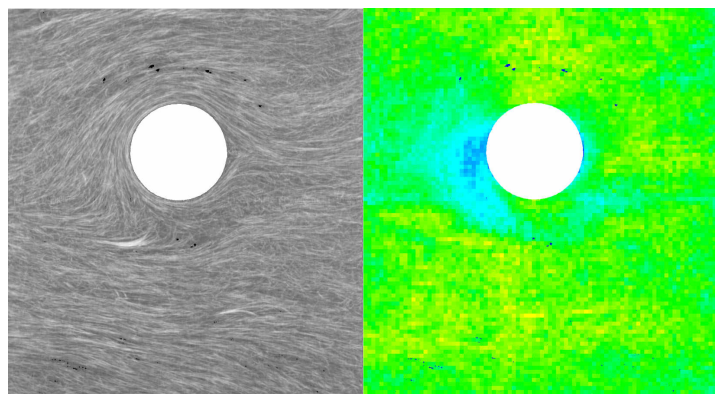

(d) A4, center layer

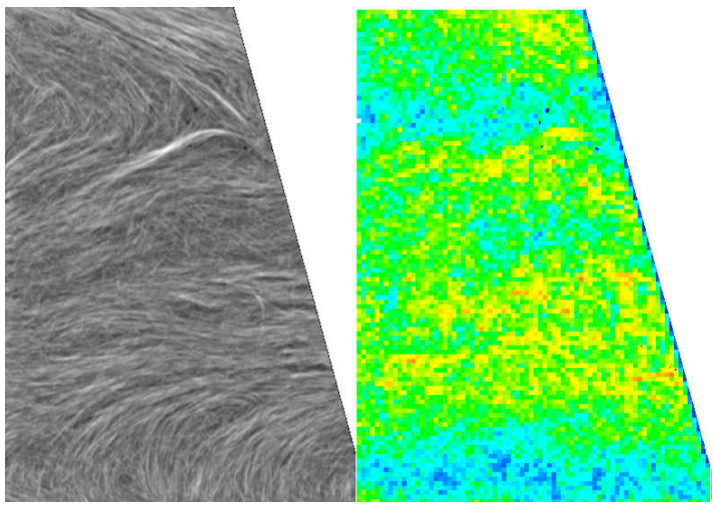

(f) sub-sample of A3.1, center layer

Figure 6: Orientation estimation results for three regions marked yellow in Figures 4 5. $2 \mathrm{D}$ slices from the original $3 \mathrm{D}$ images and from the image holding the corresponding computed local tensor component $a_{y y}$ are shown next to each other. The orientation tensors are computed in cubic sub-volumes of $(218 \mu \mathrm{m})^{3}$ for all data sets.

Figure 13 for simulation and $\mu \mathrm{CT}$ data respectively. The tetrahedral Moldflow mesh can be seen in Figure 13 along with three tetrahedra for which we calculated orientation tensors for comparison with the results from $\mu \mathrm{CT}$.
The used mesh size is app. $2 \mathrm{~mm}$ (element edge length in-plane). 12 layers are used over the part thickness (out-of-plane) for the calculations. The entire carrier has around 170000 elements using Dual Domain mesh. For the orientation calculation, 


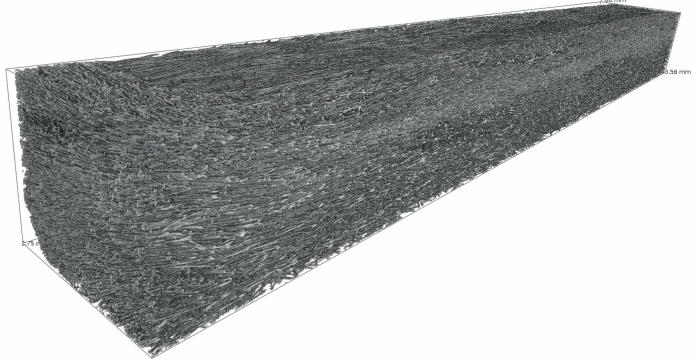

Figure 7: A3.3, cropped to the plate region, resulting in a $1200 \times 8832 \times 1000$ voxel sub-volume. The area closest to the reader is close to an edge where fibers are forced to bend.

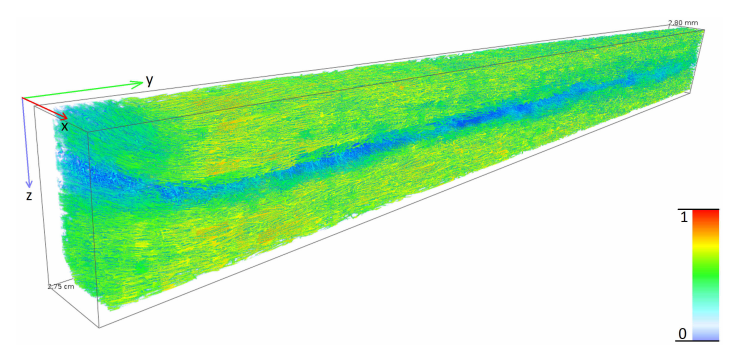

Figure 8: Clipped visualization of the evaluated area. The orientation tensor component $a_{y y}$ is shown in the analyzed sub-volumes of edge length 65 pixels (about $200 \mu \mathrm{m}$ ) leading to a grid of $17 \times 135 \times 13$ cubes. Averaged over the whole analyzed volume, the orientation tensor diagonal components are $a_{x x}=0.20, a_{y y}=0.63, a_{z z}=$ 0.15. The anisotropy index is 0.75 and the mean fiber direction $(-0.00,-0.99,-0.01)^{T}$.

the Moldflow Rotational Diffusion model is used, see e. g. [47. Its parameters - fiber interaction coefficient $\mathrm{Ci}$ and coefficients of asymmetry $D 1, D 2$ and $D 3$ - are set as "automatic calculations". That is, the default values $D 1=1.0, D 2=0.8, D 3=0.15$ are

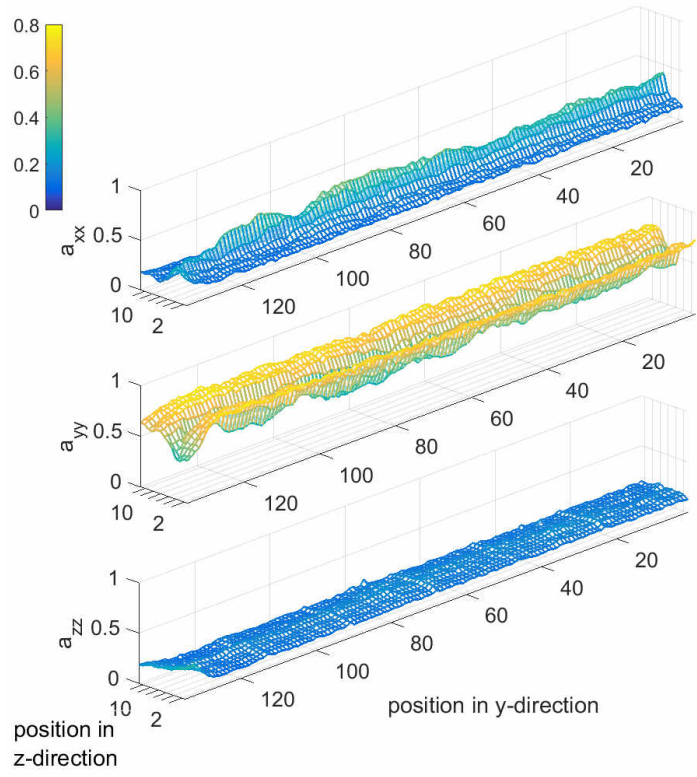

Figure 9: Orientation tensor diagonal components for the RoI scan A3.3. The mesh is based on the analysis in $135 \times 13$ sub-volumes, where the results are averaged over 17 sub-volumes along the $\mathrm{x}$-axis.

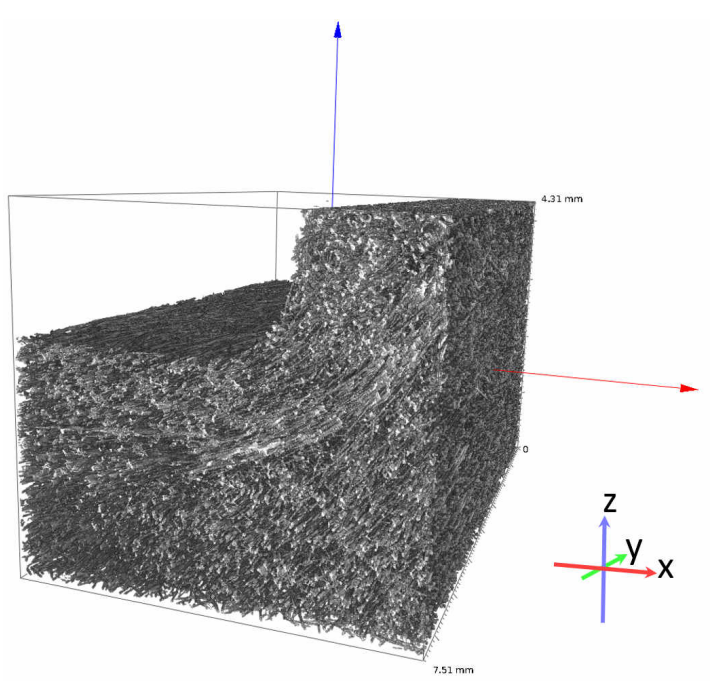

Figure 10: Volume rendering of the subvolume used for the comparison in Figure 11 

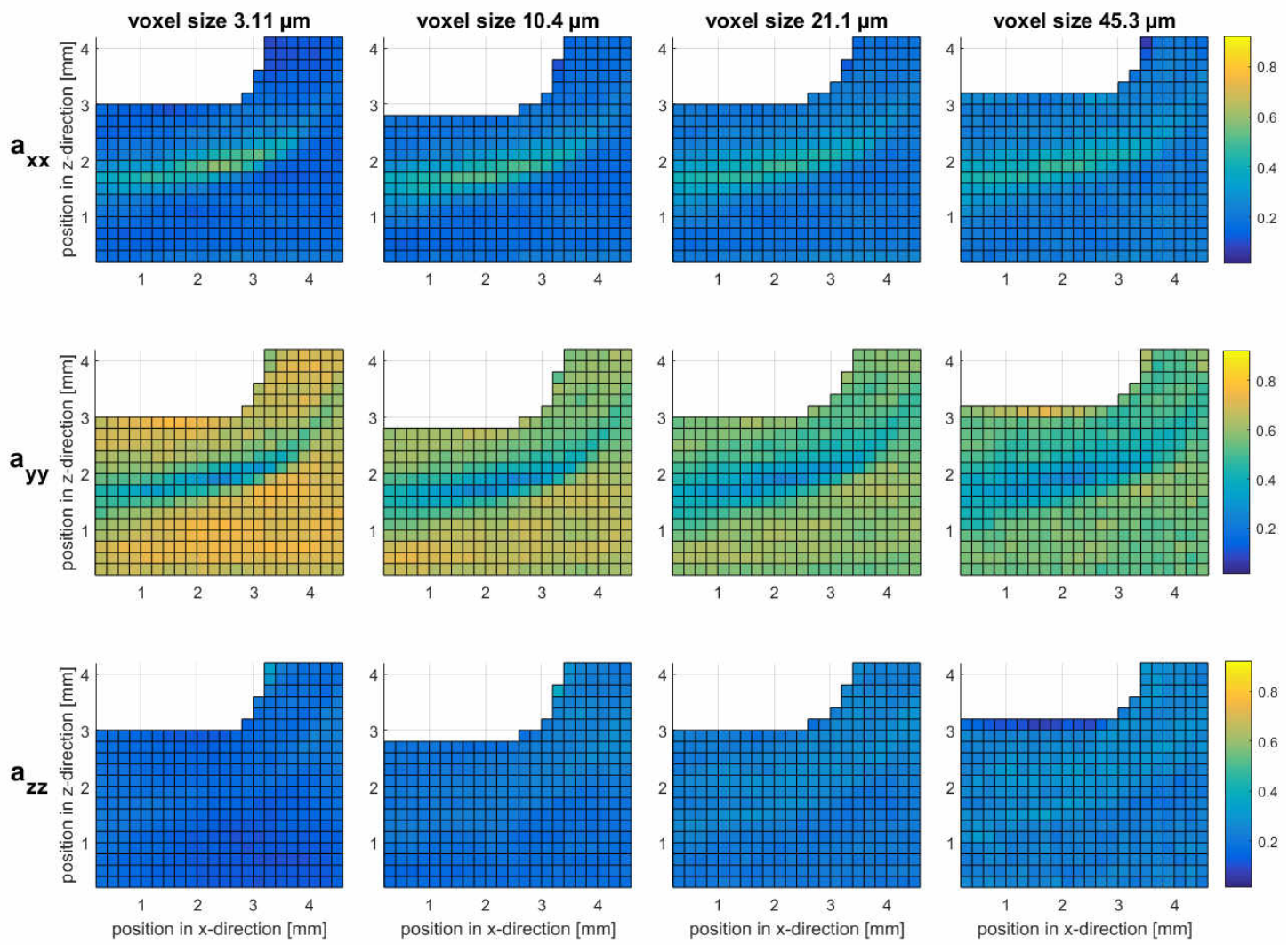

Figure 11: Comparison of local results for varying voxel sizes: Orientation tensor diagonal components for the RoI scan, color coded. A volume rendering of the corresponding sub-volume is shown in Figure 10.

used. The $\mu \mathrm{CT}$ data is processed in a way to fit the grid of simulated data. The plate thickness is subdivided in 12 cuboidal subvolumes. The orientation results are obtained by averaging along $\mathrm{x}$ - and $\mathrm{y}$-axis (in plane).

Figure 14 shows the three diagonal tensor elements $a_{x x}, a_{y y}$, and $a_{z z}$ from both the simulation and the measurement. The components $a_{x x}$ and $a_{y y}$ agree qualitatively well with $a_{x x}$ peaks in the central layer and decreasing to the outside and $a_{y y}$ showing the opposite behavior for both methods. The $a_{z z}$ component however is more pronounced in some measurement points in the simula- tion while it is almost negligible throughout in the measurement with a slight exception around the center of $\mathrm{p}_{3}$. In particular the $a_{y y}$ and $a_{z z}$ components feature kinks in the central layer that are neither explained by standard flow dynamics nor backed by the $\mu \mathrm{CT}$ measurements.

\section{Conclusion}

Based on an off-the-shelf part from the automotive industry, we showed that RoI CT is readily applicable to large FRP components provided that the CT scanner has the required motor axes and degrees of freedom. 


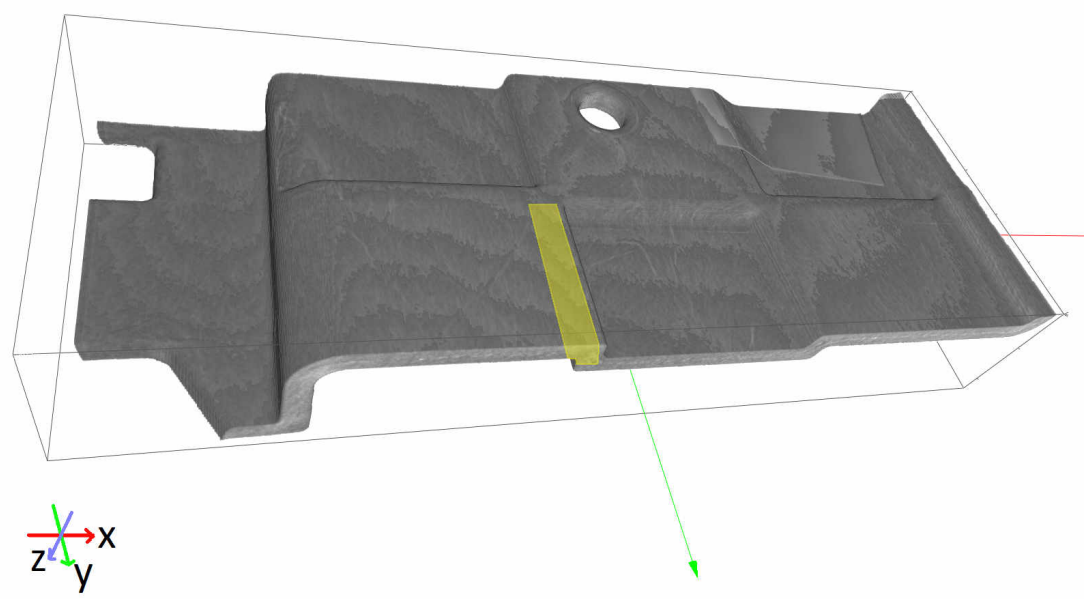

Figure 12: Visualization of a coarse CT scan around RoI A3.3 (marked yellow) that has been used for injection simulation.

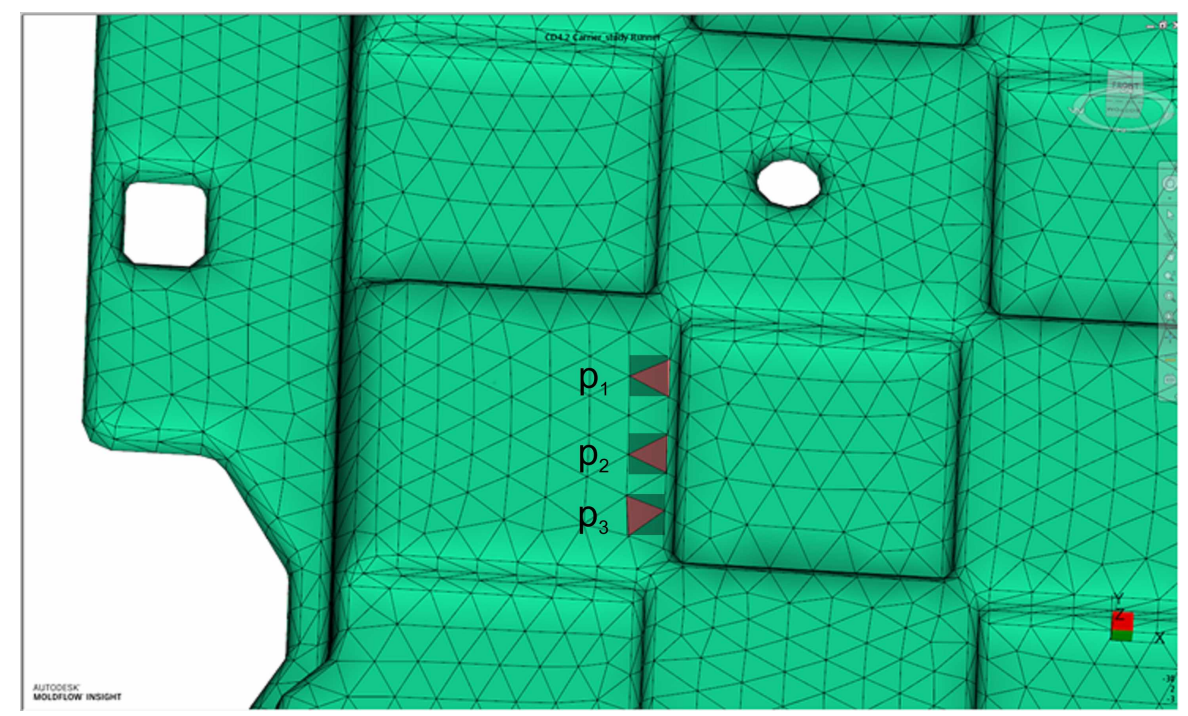

Figure 13: Mesh of the area around A3.3 that has been used for injection simulation in the software Autodesk Moldflow Insight 2017. $\mathrm{p}_{1}, \mathrm{p}_{2}$ and $\mathrm{p}_{3}$ mark the positions where the simulation results have been extracted. The simulated orientation tensor is averaged in the marked triangle, whereas the analysis based on the CT data averages in cubes.

We scanned an injection molded automotive glass FRP component that is $0.9 \mathrm{~m}$ long, $0.35 \mathrm{~m}$ wide, and has a wall thickness

of $2 \mathrm{~mm}$.

It is commonly assumed that a single fiber has to be sampled by at least $2-4$ 

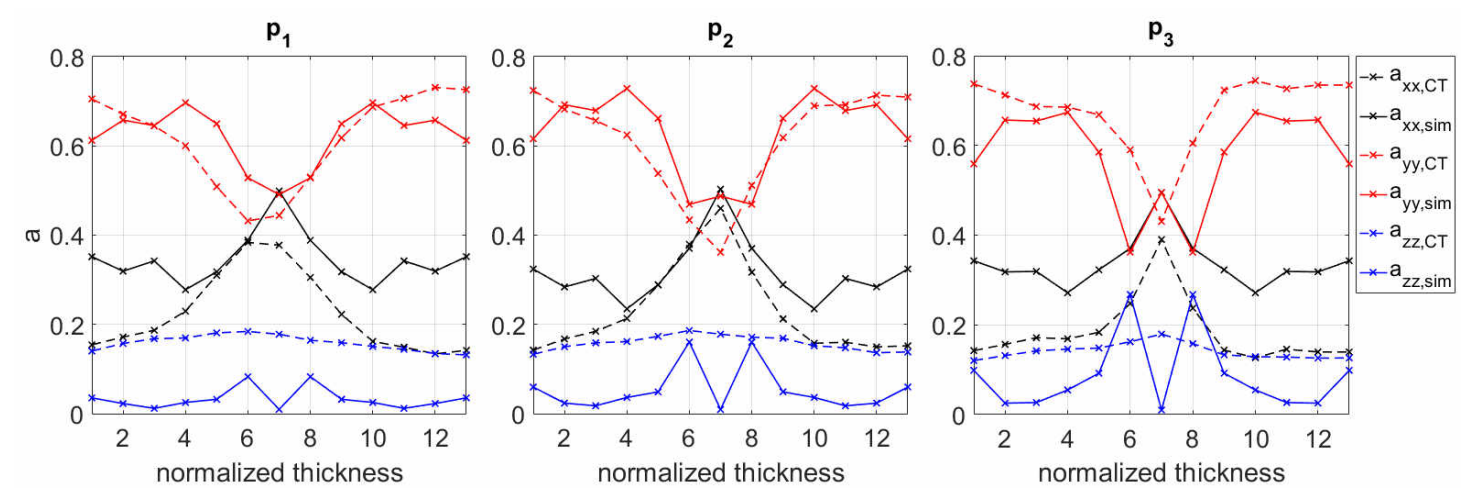

Figure 14: Comparison of simulated and CT image data based orientation tensors for region A3.3.

voxels per diameter, see the corresponding discussion in the Introduction. We clearly proved this assumption to be false. Sampling the fiber diameter by less than a voxel still allows for $3 \mathrm{D}$ orientation analysis in each voxel predominantly representing the fiber phase. The analysis method of choice gains local orientation information in each voxel from the gray value curvature captured by the Hesse matrix. The results are stabilized by averaging the 2 nd order orientation tensor over small sub-volumes. By this method we achieved good and consistent results for voxel sizes between $45 \mu \mathrm{m}$ and $3 \mu \mathrm{m}$ for the same material. Measuring the local orientation of individual fibers would require a much higher spatial sampling and therefore impose a much smaller FoV.

The 3D image analysis reveals strong anisotropy in the local fiber orientation. For all RoI except the spur shaped A2, the injection direction $\mathrm{y}$ is as expected the preferred one, see Table 2. Local effects like the typical thickness-dependent changes in the mean fiber orientation caused by flow turbulences in the central layer are captured, regardless the image quality, see Figures 6 and 11. Caution is however advised when it comes to a quantitative comparison as blurred structural information induces a bias of the orientation analysis results towards isotropy, see the right column of Figure 11. Comparing the analysis results for A3.1 and A3.2, see Table 2, shows that nominal resolution is however not the decisive parameter here.

For the best resolved RoI $\mu \mathrm{CT}$ scan A3.3, the local fiber orientations observed in the RoI CT data can be compared to those derived by injection molding simulation (Figure 14. Although deviating quantitatively, the results do agree qualitatively and particularly well for the dominating tensor component. This proves clearly that the measured orientation results can be used to validate simulation results. Thus RoI $\mu \mathrm{CT}$ combined with 3D image analysis as applied here, enables truly non-destructive 3D micro-structure characterization for large FRP components.

To summarize, we proved RoI $\mu \mathrm{CT}$ to be a potential standard tool for local fiber orientation analysis in glass fiber-reinforced automotive parts. Also, we applied successfully the proposed RoI scanning technique in combination with the orientation analysis method to carbon FRP in [54]. Our 
results are very encouraging and suggest that the method is applicable to both short and long fiber reinforced composites. Unlike methods which try to find single individual fibers and which therefore require a very high resolution and consequently cover a very small measurement volume, our method allows the extension of the latter to some 2000 times the fiber diameter, hence $3 \mathrm{~cm}$ for $15 \mu \mathrm{m}$ thick glass fibers.

\section{Acknowledgement}

This work has been supported by the Fraunhofer Society under project MEF 3D Volant. DD has been supported by the German Federal Ministry of Education and Research (BMBF) under grant 05M13RCA.

\section{References}

[1] S. Advani and C. Tucker. The use of tensors to describe and predict fiber orientation in short fiber composites. Journal of Rheology, 31(8):751-784, 1987.

[2] H. Altendorf and D. Jeulin. 3d directional mathematical morphology for analysis of fiber orientations. Image Analysis and Stereology, 28(3):143153, 2009.

[3] H. Altendorf and D. Jeulin. Fiber separation from local orientation and probability maps. In Abstract book of the ISMM '09. 9th Int. Symp. Mathematical Morphology, 2009.

[4] H. Andrä, D. Dobrovolskij, K. Schladitz, S. Staub, and R. Müller. Modelling of geometrical microstructures and mechanical behaviour of constituents. In S. Diebels and
S. Rjasanow, editors, Multi-scale Simulation of Composite Materials: Results from the Project MuSiKo, pages 31-56. Springer, Berlin Heidelberg, 2019 .

[5] H. Andrä, M. Gurka, M. Kabel, S. Nissle, C. Redenbach, K. Schladitz, and O. Wirjadi. Geometric and mechanical modeling of fiber-reinforced composites. In D. Bernard, J. Buffière, T. Pollock, H. Poulsen, A. Rollett, and M. Uchic, editors, Proceedings of the 2nd International Congress on $3 \mathrm{D} \mathrm{Ma-}$ terials Science, pages 35-40. Springer, Cham, 2014.

[6] A. Ayadi, H. Nouri, S. Guessasma, and F. Roger. Determination of orthotropic properties of glass fibre reinforced thermoplastics using x-ray tomography and multiscale finite element computation. Composite Structures, 136:635 - 649, 2016.

[7] E. Belmonte, M. De Monte, T. Riedel, and M. Quaresimin. Local microstructure and stress distributions at the crack initiation site in a short fiber reinforced polyamide under fatigue loading. Polymer Testing, 54:250-259, 2016.

[8] A. Bernasconi, F. Cosmi, and P. Hine. Analysis of fibre orientation distribution in short fibre reinforced polymers: A comparison between optical and tomographic methods. Composites Science and Technology, 72(16):20022008, 2012.

[9] D. Bull, S. Spearing, I. Sinclair, and L. Helfen. Three-dimensional assessment of low velocity impact damage in particle toughened composite laminates using micro-focus X-ray com- 
puted tomography and synchrotron radiation laminography. Composites Part A: Applied Science and Manufacturing, 52:62-69, 2013.

[10] M. De Monte, E. Moosbrugger, and M. Quaresimin. Influence of temperature and thickness on the off-axis behaviour of short glass fibre reinforced polyamide 6.6-cyclic loading. Composites Part A: Applied Science and Manufacturing, 41(10):1368-1379, 2010.

[11] M. De Monte, E. Moosbrugger, and M. Quaresimin. Influence of temperature and thickness on the offaxis behaviour of short glass fibre reinforced polyamide 6.6-quasistatic loading. Composites Part A: Applied Science and Manufacturing, 41(7):859-871, 2010.

[12] B. R. Denos, D. E. Sommer, A. J. Favaloro, R. B. Pipes, and W. B. Avery. Fiber orientation measurement from mesoscale ct scans of prepreg platelet molded composites. Composites Part A: Applied Science and Manufacturing, 114:241 - 249, 2018.

[13] D. Dobrovolskij, J. Persch, K. Schladitz, and G. Steidl. Structure detection with second order Riesz transform. Image Analysis 8 Stereology, 38(1):107119, 2019 .

[14] C. Eberhardt and A. Clarke. Fibreorientation measurements in shortglass-fibre composites. part i: automated, high-angular-resolution measurement by confocal microscopy. Composites Science and Technology, 61(10):1389-1400, 2001.

[15] C. Eberhardt and A. Clarke. Automated reconstruction of curvilinear fi- bres from 3D datasets acquired by Xray microtomography. Journal of $\mathrm{Mi}$ croscopy, 206(1):41-53, 2002.

[16] D. Eberly, R. Gardner, B. Morse, S. Pizer, and C. Scharlach. Ridges for image analysis. J. Mathematical Imaging and Vision, 4(4):353-373, 1994.

[17] M. J. Emerson, K. M. Jespersen, A. B. Dahl, K. Conradsen, and L. P. Mikkelsen. Individual fibre segmentation from 3D X-ray computed tomography for characterising the fibre orientation in unidirectional composite materials. Composites Part A: Applied Science and Manufacturing, 97:83 92, 2017.

[18] S. Fliegener, T. Kennerknecht, and M. Kabel. Investigations into the damage mechanisms of glass fiber reinforced polypropylene based on micro specimens and precise models of their microstructure. Composites Part B: Engineering, 112:327 - 343, 2017.

[19] S. C. Garcea, Y. Wang, and P. Withers. X-ray computed tomography of polymer composites. Composites Science and Technology, 156:305 - 319, 2018.

[20] C. Hannesschläger, V. Revol, B. Plank, D. Salaberger, and J. Kastner. Fibre structure characterisation of injection moulded short fibre-reinforced polymers by X-ray scatter dark field tomography. Case Studies in Nondestructive Testing and Evaluation, 3:34 - 41, 2015.

[21] F. Heieck, F. Hermann, P. Middendorf, and K. Schladitz. Influence of the cover factor of $2 \mathrm{~d}$ biaxial and triaxial 
braided carbon composites on their inplane mechanical properties. Composite Structures, 163:114 - 122, 2017.

[22] P. Hine, R. Duckett, P. Caton-Rose, and P. Coates. Fibre orientation structures and their effect on crack resistance of injection moulded transverse ribbed plate. Plastics, rubber and composites, 33(1):43-53, 2004.

[23] M. Krause, J. Hausherr, B. Burgeth, C. Herrmann, and W. Krenkel. Determination of the fibre orientation in composites using the structure tensor and local X-ray transform. J. Material Science, 45(4):888-896, 2010.

[24] M. Kronenberger, K. Schladitz, B. Hamann, and H. Hagen. Fiber segmentation in crack regions of steel fiber reinforced concrete using principal curvature. Image Analysis \&6 Stereology, 37(2):127-137, 2018.

[25] J. Mecke and W. Nagel. Stationäre räumliche Faserprozesse und ihre Schnittzahlrosen. Elektron. Informationsverarb. Kyb., 16:475-483, 1980.

[26] H. Miled, L. Silva, J. F. Agassant, and T. Coupez. Numerical simulation of fiber orientation and resulting thermo-elastic behavior in reinforced thermo-plastics. In Mechanical Response of Composites. Computational Methods in Applied Sciences, volume 10, pages 293-313, Dordrecht, 2008. Springer Netherlands.

[27] T. Mishurova, N. Rachmatulin, P. Fontana, T. Oesch, G. Bruno, E. Radi, and I. Sevostianov. Evaluation of the probability density of inhomogeneous fiber orientations by computed tomography and its application to the calculation of the effective properties of a fiber-reinforced composite. International Journal of Engineering Science, 122:14-29, 2018.

[28] B. Mlekusch, E. Lehner, and W. Geymayer. Fibre orientation in shortfibre-reinforced thermoplastics i. contrast enhancement for image analysis. Composites science and technology, 59(4):543-545, 1999.

[29] W. Nagel. Dünne Schnitte von stationären räumlichen Faserprozessen. Math. Operationsforsch. Stat., Ser. Stat, 14:569-576, 1983.

[30] J. Ohser and K. Schladitz. 3d Images of Materials Structures - Processing and Analysis. Wiley VCH, Weinheim, 2009.

[31] N. Otsu. A threshold selection method from gray level histograms. IEEE Trans. Systems, Man and Cybernetics, 9:62-66, Mar. 1979.

[32] P. Pinter, B. Bertram, and K. A. Weidenmann. Novel Method for the Determination of Fibre Length Distributions from $\mu \mathrm{CT}$-data. Conference on Industrial Computed Tomography (iCT), 2016.

[33] P. Pinter, S. Dietrich, B. Bertram, L. Kehrer, P. Elsner, and K. Weidenmann. Comparison and error estimation of $3 \mathrm{~d}$ fibre orientation analysis of computed tomography image data for fibre reinforced composites. NDT $\& E$ International, 95:26 - 35, 2018.

[34] F. Prade, F. Schaff, S. Senck, P. Meyer, J. Mohr, J. Kastner, and F. Pfeiffer. Nondestructive characterization 
of fiber orientation in short fiber reinforced polymer composites with X-ray vector radiography. NDT $\& E$ International, 86:65-72, 2017.

[35] C. Redenbach, A. Rack, K. Schladitz, O. Wirjadi, and M. Godehardt. Beyond imaging: on the quantitative analysis of tomographic volume data. International Journal of Materials Research, 2:217-227, 2012.

[36] G. Requena, G. Fiedler, B. Seiser, P. Degischer, M. Di Michiel, and T. Buslaps. 3D-quantification of the distribution of continuous fibres in unidirectionally reinforced composites. Composites Part A: Applied Science and Manufacturing, 40(2):152163, 2009.

[37] K. Robb, O. Wirjadi, and K. Schladitz. Fiber orientation estimation from 3d image data: Practical algorithms, visualization, and interpretation. In Proc. 7th Int. Conf. Hybrid Intelligent Systems, pages 320-325, Kaiserslautern, Germany, Sept 17-19 2007.

[38] D. Salaberger, K. A. Kannappan, J. Kastner, J. Reussner, and T. Auinger. Evaluation of computed tomography data from fibre reinforced polymers to determine fibre length distribution. International Polymer Processing, 26(3):283-291, 2011.

[39] K. Sandau and J. Ohser. The chord length transform and the segmentation of crossing fibres. J. Microscopy, 226(1):43-53, 2007.

[40] K. Schladitz, A. Büter, M. Godehardt, O. Wirjadi, J. Fleckenstein, T. Gerster, U. Hassler, K. Jaschek, M. Maisl, U. Maisl, S. Mohr, U. Netzelmann,
T. Potyra, and M. Steinhauser. Nondestructive characterization of fiber orientation in reinforced SMC as input for simulation based design. Composite Structures, 160:195 - 203, 2017.

[41] S. Schmidt, D. Niedziela, K. Steiner, and J. Zausch. CoRheoS: Multiphysics solver framework and simulation infrastructure for complex rheologies. In Proceedings of Nafems World Congress 2013, Salzburg, Austria, 2013.

[42] R. Schneider and W. Weil. Stochastic and Integral Geometry. Probability and Its Applications. Springer, Heidelberg, 2008.

[43] R. Sencu, Z. Yang, Y. Wang, P. Withers, C. Rau, A. Parson, and C. Soutis. Generation of micro-scale finite element models from synchrotron X-ray $\mathrm{CT}$ images for multidirectional carbon fibre reinforced composites. Composites Part A: Applied Science and Manufacturing, 91:85-95, 2016.

[44] H. Shen, S. Nutt, and D. Hull. Direct observation and measurement of fiber architecture in short fiber-polymer composite foam through micro-ct imaging. Composites Science and Technology, 64(13):2113 - 2120, 2004.

[45] D. Stoyan, W. S. Kendall, and J. Mecke. Stochastic Geometry and Its Applications. Wiley, Chichester, 2nd edition, 1995.

[46] M. Teßmann, S. Mohr, S. Gayetskyy, U. Haßler, R. Hanke, and G. Greiner. Automatic Determination of FiberLength Distribution in Composite Material Using 3D CT Data. EURASIP Journal on Advances in Signal Processing, 2010(1):545030, 2010. 
[47] H.-C. Tseng, R.-Y. Chang, and C.-H. Hsu. Comparison of recent fiber orientation models in injection molding simulation of fiber-reinforced composites. Journal of Thermoplastic Composite Materials, 2018. https://doi. org/10.1177/0892705718804599.

[48] J. Viguié, P. Latil, L. Orgéas, P. Dumont, S. R. du Roscoat, J.-F. Bloch, C. Marulier, and O. Guiraud. Finding fibres and their contacts within $3 \mathrm{~d}$ images of disordered fibrous media. Composites Science and Technology, 89:202 - 210, 2013.

[49] J. Weissenböck, A. Amirkhanov, W. Li, A. Reh, A. Amirkhanov, E. Gröller, J. Kastner, and C. Heinzl. Fiberscout: an interactive tool for exploring and analyzing fiber reinforced polymers. In 2014 IEEE Pacific Visualization Symposium, pages 153-160. IEEE, 2014.

[50] O. Wirjadi, M. Godehardt, K. Schladitz, B. Wagner, A. Rack, M. Gurka, S. Nissle, and A. Noll. Characterization of multilayer structures in fiber reinforced polymer employing synchrotron and laboratory X-ray CT. International Journal of Materials Research, 105(7):645-654, 2014.

[51] O. Wirjadi, K. Schladitz, P. Easwaran, and J. Ohser. Estimating fibre direction distributions of reinforced composites from tomographic images. Image Analysis and Stereology, 35(3):167-179, 2016.

[52] O. Wirjadi, K. Schladitz, A. Rack, and T. Breuel. Applications of anisotropic image filters for computing $2 \mathrm{~d}$ and 3d-fiber orientations. In V. Capasso, G. Aletti, and A. Micheletti, editors,
Stereology and Image Analysis - 10th European Congress of ISS, pages 107112, Milan, Italy, June 2009.

[53] A. Wonisch and A. Wüst. More precise part design: Accurate simulation of fiber orientation of glass fiber-reinforced plastics. Kunststoffe International, pages 80-83, 2014. WwW.kunstst of fe-international. com/894849.

[54] S. Zabler, K. Schladitz, K. Dremel, J. Graetz, and D. Dobrovolskij. Region-of-interest X-ray tomography for the non-destructive characterization of local fiber orientation in large fiber composite parts. In Key Engineering Materials, volume 809, pages 587-593. Trans Tech Publ, 2019. 